\title{
Research on bathymetry estimation by Worldview-2 based with the semi-analytical model
}

\author{
SHENG Lin ${ }^{a, c}$, BAI Jie ${ }^{a,{ }^{*}}$, ZHOU Gao-wei ${ }^{a, b}$, ZHAO Yali,LI ${ }^{a}$, Ying-cheng $^{a, b}$ \\ $a$ *.China TOPRS Technology Co., Ltd, Beijing 100039, China, 13811199372@126.com; \\ b. Chinese Academy of Surveying and Mapping, Beijing 100039, China,casm2012@163.com; \\ c. Henan Polytechnic University School of Surveying and Land Information Engineering, Henan Jiaozuo 454000, \\ China,shenglin417@163.com;
}

Key words: Bathymetry Estimation; Semi-analytical Model; Genetic Algorithm; Remote Sensing

\begin{abstract}
South Sea Islands of China are far away from the mainland, the reefs takes more than 95\% of south sea, and most reefs scatter over interested dispute sensitive area. Thus, the methods of obtaining the reefs bathymetry accurately are urgent to be developed. Common used method, including sonar, airborne laser and remote sensing estimation, are limited by the long distance, large area and sensitive location. Remote sensing data provides an effective way for bathymetry estimation without touching over large area, by the relationship between spectrum information and bathymetry. Aimed at the water quality of the south sea of China, our paper develops a bathymetry estimation method without measured water depth. Firstly the semi-analytical optimization model of the theoretical interpretation models has been studied based on the genetic algorithm to optimize the model. Meanwhile, OpenMP parallel computing algorithm has been introduced to greatly increase the speed of the semi-analytical optimization model. One island of south sea in China is selected as our study area, the measured water depth are used to evaluate the accuracy of bathymetry estimation from Worldview-2 multispectral images. The results show that: the semi-analytical optimization model based on genetic algorithm has good results in our study area;the accuracy of estimated bathymetry in the 0-20 meters shallow water area is accepted.Semi-analytical optimization model based on genetic algorithm solves the problem of the bathymetry estimation without water depth measurement. Generally, our paper provides a new bathymetry estimation method for the sensitive reefs far away from mainland.
\end{abstract}

\section{INTRODUCTION}

Shallow bathymetry around the sea reefs or islands is important to the terrain elements, and also is the premise of marine work.Commonly used bathymetry estimation models are the combination of semi-empiricaland semi-theoretical model, depending on certain theoretical and empirical parameters. However the application and accuracy of these models are limited by the usable and precision of measured depth data before model construction, which is difficult to obtainfor the islands or reefs far away from the mainland.Therefore the theoretical models based on the radiation field distribution of sea water are selected to estimate the bathymetry by remote sensing data in this paper. Currently, because of many uncertain parameters in theoretical models are complex and

*Bai Jie. Email: 13811199372@126.com difficult to be fixed, related researches are few.Based on double layer approximation model, Zyzenga (1978) derived the surface remote-sensing reflectance for the shallow waters, and then obtained the bathymetry, but the model is only a theoretical experiment.Based on the simplified model of the radiative transfer equation by Jupp (1988) and Bierwirth (1993), Zhou Yan (2011) established the direct inverse radiative transfer model. But the value of the attenuation coefficient in this model was directly achieved by the quasi-analytical algorithm,resultingthe increment of waterreflectioninformation and decreasing the model accuracy. So it is necessary to study the theoretical bathymetry estimation model without measured water depths. In this paper, by referencing ocean biomass estimation in the ocean color remote sensing, the semi-analytical model is establishedto estimate the bathymetry. 


\section{STUDY AREA AND DATA SOURCES}

East Island locates in the east of the Xuande atoll of Paracel Islands and is selected as our study area, which is 50 kilometers apart away from Yongxing Island.

Worldview-2 remote sensing image is used for bathymetry estimation. The acquisition time is September 20, 2012; with 2 meters spatial resolution. Some details are shown in Table 1. The measured water depth data has been acquired between May and August 2011.

Table 1 Band characteristics of Worldview-2

\begin{tabular}{rcc}
\hline BAND & $\begin{array}{c}\text { Central } \\
\text { Wavelength }(\mathrm{m})\end{array}$ & $\begin{array}{c}\text { Spatial } \\
\text { Resolution(m) }\end{array}$ \\
\hline Pan & 628 & 0.5 \\
Coastal & 425 & 2 \\
Blue & 480 & 2 \\
Green & 545 & 2 \\
Yellow & 605 & 2 \\
Red & 660 & 2 \\
Red edge & 725 & 2 \\
Near-IR1 & 833 & 2 \\
Near-IR2 & 950 & 2 \\
\hline
\end{tabular}

\section{MODELING AND IMPLEMENTATION}

\subsection{Image Pre-processing}

Based on UVA DOMimage and header file from Worldview-2 image, geometric correction, radiation calibration and atmospheric correction has been carried out for original image. The values of $\mathrm{DN}$ are transformed to reflectance, the influence of atmospheric scattering and absorption are also corrected.

\subsection{Water Information Extraction}

The land information in the image should be removed before bathymetry estimation model constructing, which is used to generate a new image only containing water area. In our study, the image of Near-IR1 band is applied in edge detection with Canny algorithm. After series of experiment, the value of the double threshold in the Canny algorithm has been determined, with the lower threshold is 20 and the higher one is 1000 . The extracted edge line of our study area is showed in Figure1. In order to verify the effect of edge detection, the edge has been overlapped on the original image, which is also shown in Figure2.

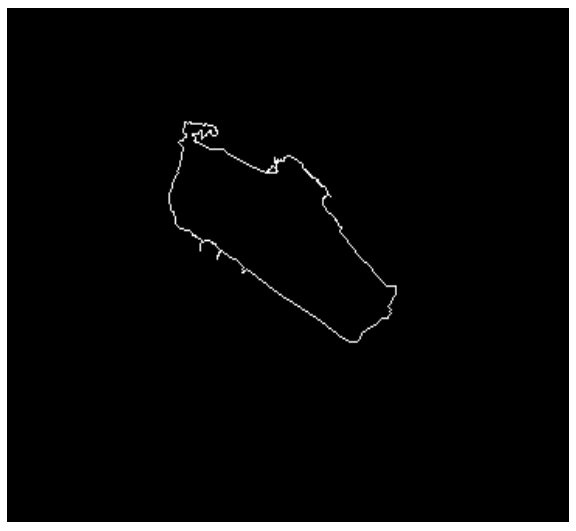

Figure 1 Result of extracting edge line by Canny algorithm

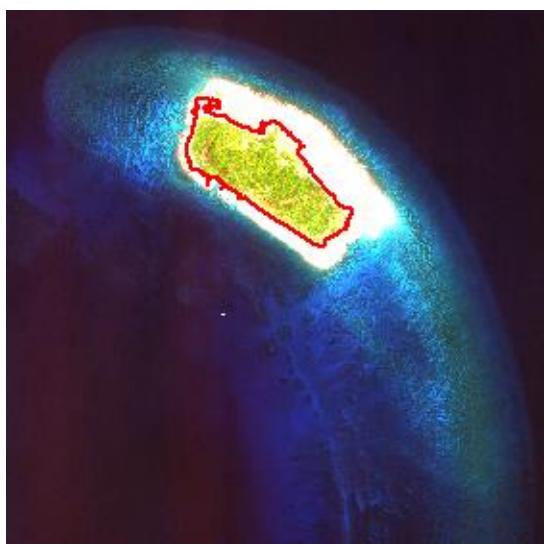

Figure 2 Overlapping of estimated edge and original image

The results show the edge is basically consistent with the land edge. And then the edge line image has been masked by assigning the pixel values of land inside the edge to 0 and others to 1 . Finally, the mask is multiplied with the corrected image to extract water information

\subsection{Semi-analytical Model Construction}

By referencing the semi-analytical model of ocean biomass inversing from the ocean color remote sensing, we established the bathymetry estimation model. As for shallow waters, the relationship of subsurfaceremote-sensing reflectance and abovesurface remote-sensing reflectance has been firstly determined based on simulated data (Lee, 1999). 


$$
R_{r s}=\frac{0.5 r_{r s}}{1-1.5 r_{r s}}
$$

Where, $R_{r s}$ is above-surface remote-sensing reflectance; $r_{r s}$ is subsurface remote-sensing reflectance.Then the water-leaving radiance model has been corrected by adding subsurface solar zenith angel, viewing azimuth angel and subsurface viewing angel. So the semi-analytical model for $r_{r s}$ is

$$
\begin{aligned}
& r_{r s} \approx r_{r s}^{d p}\left(1-\exp \left\{-\left[-\frac{1}{\cos \left(\theta_{w}\right)}+\frac{D_{u}^{C}}{\cos (\theta)}\right] \kappa H\right\}\right) \\
& +\frac{1}{\pi} \rho \exp \left\{-\left[-\frac{1}{\cos \left(\theta_{w}\right)}+\frac{D_{u}^{B}}{\cos (\theta)}\right] \kappa H\right\}
\end{aligned}
$$

Where

$$
\begin{aligned}
& \left\{\begin{array}{c}
r_{r s}^{d p} \approx(0.084+0.170 u) u \\
D_{u}^{C} \approx 1.03(1+2.4 u)^{0.5} \\
D_{u}^{B} \approx 1.04(1+5.4 u)^{0.5}
\end{array}\right. \\
& u=b_{b} /\left(a+b_{b}\right) \quad \kappa=a+b_{b}
\end{aligned}
$$

Where $\theta_{w}$ is subsurface solar zenith angel; $\theta$ is subsurface viewing angel; $\kappa$ is an attenuation coefficient; $H$ is bathymetry; $a$ is total absorption coefficient of water; $b_{b}$ is total backscattering coefficient; $\rho$ is bottom albedo. But in order to prevent pathological inversion, the original model has been parameterized by bio-optical model (Lee, 1998).

Wherein the total water absorption coefficient $a(\lambda)$ is

$$
\begin{gathered}
a(\lambda)=a_{w}(\lambda)+a_{\phi}(\lambda)+a_{g}(\lambda) \\
\left\{\begin{array}{c}
a_{\phi}(\lambda)=\left[a_{0}(\lambda)+a_{1}(\lambda) \ln (P)\right] P \\
P=a_{\phi}(440)
\end{array}\right. \\
\left\{\begin{array}{c}
a_{g}(\lambda)=G \exp [-S(\lambda-440)] \\
G=a_{g}(440)
\end{array}\right.
\end{gathered}
$$

Here $a_{w}(\lambda)$ is the absorption coefficient of pure water; $a_{\phi}(\lambda)$ is phytoplankton absorption coefficient; $a_{g}(\lambda)$ is the absorption coefficient of gelbstoff and detritus. Among them, $a_{0}(\lambda) a_{1}(\lambda)$ and $a_{w}(\lambda)$ are taken from empirical parameters;is the spectrum slope in the range of $0.011-0.021 \mathrm{~nm}-1 ; S$ value of $0.015 \mathrm{~nm}$ is used in the inversion process.

The total coefficient of backscattering $b_{b}(\lambda)$ is:

$$
b_{b}(\lambda)=b_{b w}(\lambda)+b_{b p}(\lambda)
$$

$$
\begin{gathered}
\left\{\begin{array}{c}
b_{b p}(\lambda)=X\left(\frac{400}{\lambda}\right)^{Y} \\
X=b_{b p}{ }^{\prime}(440)
\end{array}\right. \\
X=b_{b p}{ }^{\prime}(440) \\
=b_{b w}(440)+\varepsilon(\lambda) b_{b p}(440) \\
\left\{\begin{array}{c}
Y=3.44[1-3.17 \exp (-2.01 \chi)] \\
\chi=R_{r s}(480) / R_{r s}(545)
\end{array}\right.
\end{gathered}
$$

Here $b_{b w}(\lambda)$ is the backscattering coefficient of pure sea water; $b_{b p}(\lambda)$ is the backscattering coefficient of particles.

The bottom albedo of $\rho$ is expressed by a 550-nm-normalized albedo shape.

$$
\left\{\begin{array}{c}
\rho(\lambda)=B \rho_{\text {norm }}(\lambda) \\
B=\rho(550)
\end{array}\right.
$$

\subsection{Implementation Model based on Genetic Algorithm}

After parameterization, the parameters of $P, G, X, B, H$ determine the remote-sensing reflectance in theory to inverse bathymetry, as long as there is no less than five channels of remote sensing data. But the radiative transfer equation is still complex to solve in the situation of no adequate data, and the speed of the calculation is slow. Thus, the genetic algorithm is used to solve the parameters under the condition of lack of data sources; moreover, OpenMP parallel processing model is applied to the genetic algorithm for saving the hours of solving process.

\subsubsection{Bottom Type Determination}

The bottom sediment types of sea water can be divided into two types: sand and algae. We use the empirical formulas to distinguish between different types of sediment. If the water-leaving reflectance of pixel satisfiesequation 13, the type of plant is judged; otherwise the type of bottom sediment is seemed as sand (Lee, 2002a).

$$
\left\{\begin{array}{c}
R_{r s}(550)<0.01 \\
R_{r s}(725) / R_{r s}(660)>1.2
\end{array}\right.
$$

The water-leaving reflectance of red edge, red and green band is substituted into equation (13). The result shows that the experimental area of bottom 
sediment type is sand. Therefore the shapeof 550-nm-normalized albedo is used, as shown in Figure 3.

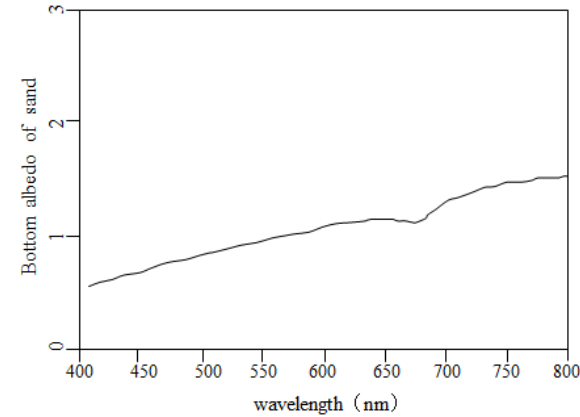

Figure3 550-nm normalized bottom albedo spectrum

\subsubsection{Parameter Calculation based on Genetic}

\section{Algorithm}

The initial parameters are assigned firstly, with genetic generation is 6000 ; population number is 50 ; crossover rate is 0.85 , mutation rate is 0.01 . Secondly, the ranges of five parameters are fixed, and the initial population from the parameters range is determined to select individuals randomly. Thirdly, the individuals of initial population are coded using real-number way. In the end, the phenomena of natural selection, breeding and genetic mutations are simulated by fitness information. That is to say, the individuals of each generation of population are carried out the selection algorithm, crossover algorithm and mutation algorithm to produce a new generation of population. This process of genetic algorithmis repeateduntil it reaches the settled generation. Just then the parameters of semi-analytical model have been calculated; meanwhile the bathymetry of shallow water is obtained. Parts of running results are shown in Figure 4.

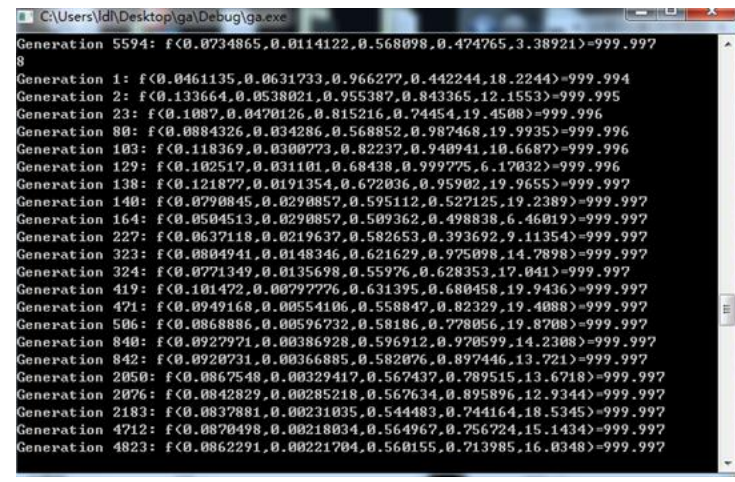

Figure 4 Parts of running results of genetic algorithm

The value before colon in each line is the number of genetic generation; and after colon is the values of five parameters of $P 、 G 、 X 、 B$ and $H$ in each generation; the end value of each line is model error of current generation. Due to the small value of error, we add 1000 to the model error just in order to facilitate comparison in the algorithm implementation.

The genetic algorithm process is time-consuming,especially for the situation of only providing the approximate rangeof parameters. Therefore OpenMP parallel processing model is applied to improve the semi-analytical model calculation of the genetic algorithm pixel by pixel, by saving several hours. The experiment of parallel acceleration suitable for"for loop", and the testing machine is configured for 8-core Intel (R) Xeon (R) processor, memory $8 \mathrm{G}$. The acceleration efficiency is compared before and after the procedure as shown in Figure 5 and Figure 6.

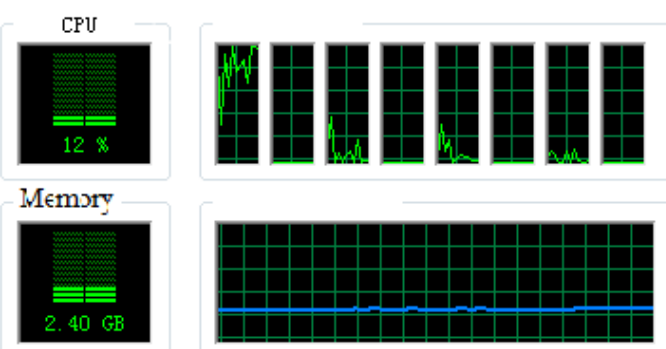

Figure 5 Acceleration efficiency before using OpenMP parallel processing model

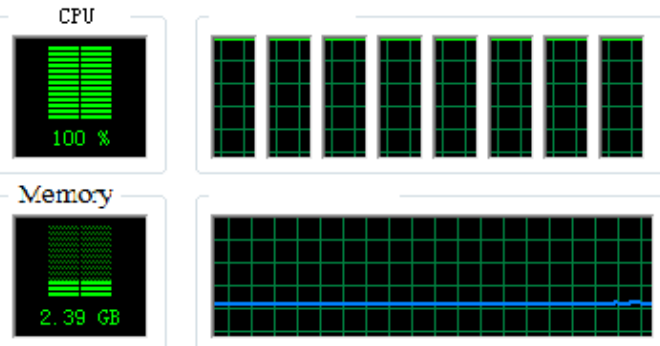

Figure 6 Acceleration efficiencyafter using OpenMP parallel processing model

After speeding up, the rate of CPU is improved from $12 \%$ to $100 \%$, making full use of multi-core processors, and enhancing the operating efficiency in the circulating level. 


\subsubsection{Results and Accuracy evaluation}

Based on measured data after the vertical datum transformation, we use the mean error and relative error as indicators to evaluate the accuracy of bathymetry estimation. The evaluation points are extracted by overlapping the measured points and the corresponding bathymetry estimation. Accuracies of establishedbathymetry inversion model are shown in Table 2 .

Table 2 Accuracies of semi-analytical bathymetry inversion model

\begin{tabular}{ccc}
\hline Bathymetry $(\mathrm{m})$ & Mean error $(\mathrm{m})$ & Relative error \\
\hline $\mathbf{0 - 5}$ & 2.32 & $49.09 \%$ \\
$\mathbf{5 - 1 0}$ & 1.54 & $17.56 \%$ \\
$\mathbf{1 0 - 2 0}$ & 1.99 & $14.38 \%$ \\
$\mathbf{2 0 - 3 0}$ & 4.12 & $50.28 \%$ \\
\hline
\end{tabular}

Table 2 shows that accuracy of semi-analytical model based on genetic algorithm of bathymetry estimation, the average error of the 0 to 30 meters is $2.99 \mathrm{~m}$, the average relative error is $35.28 \%$; error for 0 to $5 \mathrm{~m}$ water is $2.32 \mathrm{~m}$, the relative error is $49.09 \%$; the error for 5 to $10 \mathrm{~m}$ water is $1.54 \mathrm{~m}$, the relative error is $17.56 \%$, error of $10-20 \mathrm{~m}$ is 1.99 meters, the relative error is $14.38 \%$; the average error of $20-30 \mathrm{~m}$ water is $4.12 \mathrm{~m}$, the average relative error is $50.28 \%$.

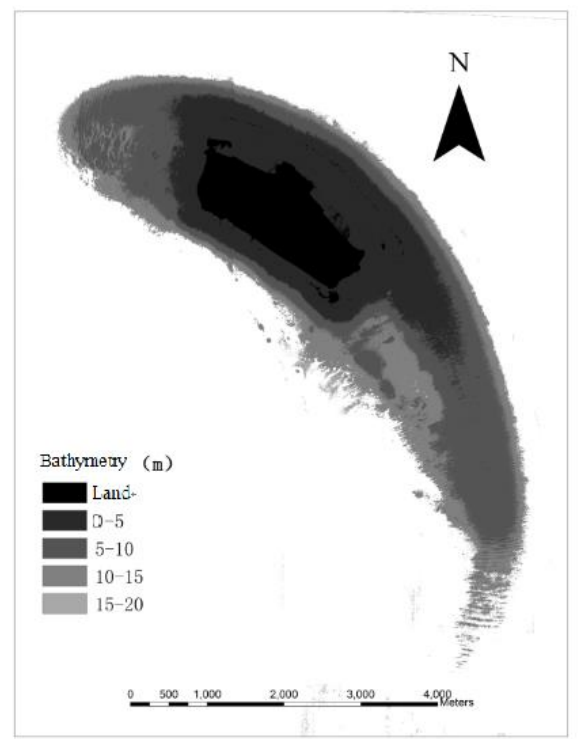

Figure7 Bathymetry estimated from semi-analytical inversion model over East Island Reef

Bathymetry within the scope of 5-20 mhas a high precision;relatively the inversion precision is lower in 0 to $5 \mathrm{~m}$ and 20 to 30 meters. Due to the inversion accuracyof the 20-30 $\mathrm{m}$ water is low; the results only contain the 0 to $20 \mathrm{~m}$ water area, as shown in Figure 7.

\section{CONCLUSIONS}

In this paper, the semi-analytical model of ocean biomass inversion is applied to bathymetry estimation with remote-sensing data. The genetic algorithm and OpenMPprogramming model for shared memory parallel processing is introduced.This method of bathymetry estimation solves the problem under the condition of lack prior knowledge, so it provides an effective way for bathymetry estimation for the sensitive islands far away from the mainland.

\section{REFERENCES}

Bierwirth P N, Lee T J, 1993a. Shallow sea floor reflectance and water depth derive by unmixing multispectral imagery. Photogrammetric Engineering and Remote Sensing, 59(3), pp, 331-338.

Jupp D L B, 1988. Background and extensions to depth of penetration (DOP) mapping in shallow coastal waters. Sympodium on Remote Sensing of the Coastal Zone. Gold Coast, Queensland.

Lee Z P, Carder K L, Arnone R A, 2002a. Deriving inherent optical properties from water color a multiband quasi-analytical algorithm for optically deep waters. Applied Optics, 41(27), pp, 5755-5772.

Lee Z P, Carder K L, Mobley C D, 1998a. Hyperspectral remote sensing for shallow waters. I. A semianalytical model. Applied Optics, 37(27), pp, 6329-6338

Lee Z P, Carder K L, Mobley C D, 1999a Hyperspectral remote sensing for shallow waters2. Deriving bottom depths and water properties by optimization. Applied Optics, 38(18), pp3831-3843

Lyzenga D R, 1978a. Passive remote sensing techniques of mapping water depth and bottom features. Applied Optics, 17(3), pp379-383.

Zhou Y, 2011a. Direct inversion method of shallow water bathymetry from Hyperion data. Journal of atmospheric and environmental optics, 6(2), pp, 112-117. 